\title{
1-loop correction to the SU(3) symmetric chiral soliton
}

\author{
H. Walliser \\ Departamento de Física, Universidade de Coimbra, P-3000 Coimbra, Portugal \\ Received 8 October 1997; revised 26 February 1998 \\ Editor: J.-P. Blaizot
}

\begin{abstract}
Masses of the SU(3) chiral soliton in tree approximation turn out at much too high energies typically around $2 \mathrm{GeV}$. It is shown that 1-loop corrections reduce this value drastically with results in the region of the empirical nucleon mass. (C) 1998 Elsevier Science B.V. All rights reserved.
\end{abstract}

PACS: 14.20.-c; 12.40.-y

Keywords: SU(3) loop

\section{Introduction}

In this short letter we discuss the effect of 1-loop corrections to the mass of the SU(3) symmetric chiral soliton. It is well-known that the soliton's tree mass turns out too high already in $\mathrm{SU}(2)$ and the SU(3) extension adds further a large kaonic rotational energy such that the situation is worsened with values which typically lie around or even above 2 $\mathrm{GeV}$. In SU(2) it was shown [1,2] that pionic 1-loop corrections are capable to reduce the too high tree mass to a reasonable number (Table 1).

The main concern of this paper is to answer the question whether the same may happen also in SU(3) where we have to start from a much larger soliton mass, and if that actually occurs how can it be in accordance with $N_{C}$ counting? In the course of this investigation we will recognize as often in soliton models the decisive role of the Wess-Zumino-Witten (WZW) term.

A detailed discussion of the $\mathrm{SU}(2)$ case is found in [2] and the SU(3) extension requires only minor changes in the formulation. The renormalisation procedure is identical to that used in chiral perturbation theory and relies on chiral counting together with dimensional regularisation which preserves chiral symmetry. Starting point is always an exact numerical solution of the classical equations of motion around which fluctuations for the 1-loop calculation are considered and the exactness of the solution guarantees that the terms linear in the fluctuations vanish. These requirements limit the applicability of the procedure considerably: in SU(3) this is the rotating hedgehog

$$
U=A U_{0} A^{\dagger}, \quad U_{0}=\left(\begin{array}{cc}
e^{i \tau \hat{r} F(r)} & \\
& 1
\end{array}\right) \quad A \in \mathrm{SU}(3)
$$

in the symmetric case $m_{K}=m_{\pi}$. Here $F(r)$ denotes the chiral angle and $A$ is a $\mathrm{SU}(3)$ rotation matrix depending on Euler angles $\alpha_{a}, a=1, \ldots, 8$. Already for weak symmetry breaking, $m_{K} \gtrsim m_{\pi}$, the ansatz (1) does not remain exact. Allowing the profile to become Euler angle dependent, $F\left(r, \alpha_{a}\right)$, improves 
the situation [3] but does not solve the problem: kaon and eta solitonic components are induced already in lowest order $\left(m_{K}^{2}-m_{\pi}^{2}\right)$. To consider fluctuations around such a SU(3) deformed object is certainly beyond present possibilities and we are therefore not in the position to calculate 1-loop in the rotator approach [4]. On the other hand, for strong symmetry breaking $m_{K} \gg m_{\pi}$ the hedgehog (1) rotating in SU(2) only, $A \in \mathrm{SU}(2)$, becomes an exact solution. However there the assumption $m_{K} \gg m_{\pi}$ is in conflict with chiral counting and the adopted regularisation scheme. Whether the standard chiral lagrangian may nevertheless be used in connection with the bound-state approach [5] will be subject of a separate investigation. At present our procedure applies only to $\mathrm{SU}(2)\left(m_{K} \rightarrow \infty\right)$ and to $\mathrm{SU}(3)$ symmetry $\left(m_{K}=\right.$ $m_{\pi}$ ) which is treated in the following. The actual nucleon mass should lie in between these two limiting cases.

\section{Formulation}

The standard chiral SU(3) lagrangian [6] expressed in terms of the matrix $U$ which contains the dynamical fields and the mass matrix $M$

$$
\begin{aligned}
\mathscr{L}= & \frac{f^{2}}{4} \operatorname{tr}\left[\partial_{\mu} U \partial^{\mu} U^{\dagger}+M\left(U+U^{\dagger}\right)\right] \\
& +\left(L_{1}+L_{2}+\frac{1}{2} L_{3}\right)\left(\operatorname{tr} \partial_{\mu} U \partial^{\mu} U^{\dagger}\right)^{2} \\
& +\frac{1}{2} L_{2} \operatorname{tr}\left(\left[U^{\dagger} \partial_{\mu} U, U^{\dagger} \partial_{\nu} U\right]\right)^{2} \\
& +\left(L_{3}+3 L_{2}\right) \\
& \times\left[\operatorname{tr} \partial_{\mu} U \partial^{\mu} U^{\dagger} \partial_{\nu} U \partial^{\nu} U^{\dagger}-\frac{1}{2}\left(\operatorname{tr} \partial_{\mu} U \partial^{\mu} U^{\dagger}\right)^{2}\right] \\
& +L_{4} \operatorname{tr} M\left(U+U^{\dagger}\right) \operatorname{tr} \partial_{\mu} U \partial^{\mu} U^{\dagger} \\
& +L_{5} \operatorname{tr}\left(U M+M U^{\dagger}\right) \partial_{\mu} U \partial^{\mu} U^{\dagger} \\
& +L_{6}\left(\operatorname{tr} M\left(U+U^{\dagger}\right)\right)^{2}+L_{7}\left(\operatorname{tr} M\left(U-U^{\dagger}\right)\right)^{2} \\
& +L_{8} \operatorname{tr}\left(M U M U+M U^{\dagger} M U^{\dagger}\right) \\
\equiv & \frac{f^{2}}{4} \operatorname{tr}\left[\partial_{\mu} U \partial^{\mu} U^{\dagger}+M\left(U+U^{\dagger}\right)\right] \\
& +\sum_{i=1}^{8} L_{i} \mathscr{L}_{i}^{(4)}
\end{aligned}
$$

comprises the familiar non-linear sigma $(\mathrm{N} \ell \sigma)$ model of (chiral order) $\mathrm{ChO} 2$ and eight terms of $\mathrm{ChO} 4$ which are relevant in the soliton sector without external fields. At scale $\mu=m_{\varrho}=770 \mathrm{MeV}$ which should provide the lagrangian in leading order $N_{C}$ [7] the renormalized low energy constants (LECs) are chosen

$$
\begin{aligned}
& L_{1}+L_{2}+\frac{1}{2} L_{3}=0, \quad L_{3}+3 L_{2}=0, \\
& L_{2}=\frac{1}{16 e^{2}}, \quad L_{4}=L_{6}=0, \\
& L_{5}=2 L_{8}=-6 L_{7}=\frac{f_{K}^{2}-f_{\pi}^{2}}{8\left(m_{K}^{2}-m_{\pi}^{2}\right)}=2.3 \cdot 10^{-3},
\end{aligned}
$$

to be in accordance with the standard values $[6,7]$ (within error bars) with one exception: $L_{2}$ has to be fixed by an effective Skyrme parameter $e=4.25$ (the standard value would correspond to $e \simeq 7$ ) in order to simulate the missing higher ChOs generated by vector mesons. A detailed justification of this choice is found in Ref. [2]. The SU(2) reduction of (2) yields exactly the lagrangian employed in that reference (and the LECs which additionally appear in SU(3) take their standard values). As a consequence the soliton as well as the pionic 1-loop results do not have to be recalculated, but may just be taken from there. It should be mentioned that although the LECs are chosen such that many of the ChO4 terms in (2) vanish at scale $\mu=m_{\rho}$ all these terms are switched on and do contribute when the scale is changed.

In the SU(3) symmetric case under consideration the mass matrix $M=m^{2} \cdot \mathbf{1}$ is diagonal and leads to identical kaon and pion masses and decay constants

$f_{K}^{2}=f_{\pi}^{2}=f^{2}+8\left(3 L_{4}+L_{5}\right) m^{2}, \quad f=91.1 \mathrm{MeV}$,

$f_{K}^{2} m_{K}^{2}=f_{\pi}^{2} m_{\pi}^{2}=f^{2} m^{2}+16\left(3 L_{6}+L_{8}\right) m^{4}$,

$m=138 \mathrm{MeV}$.

Because the symmetry breakers are absent, the nucleon mass in tree approximation 


$$
\begin{aligned}
E_{\text {tree }}= & M_{0}+\frac{J(J+1)}{2 \Theta_{\pi}} \\
& +\frac{1}{2 \Theta_{K}}\left[C_{2}-J(J+1)-\frac{N_{C}^{2}}{12}\right] \\
= & M_{0}+\frac{3}{8 \Theta_{\pi}}+\frac{N_{C}}{4 \Theta_{K}}, \quad N_{C} \text { odd }
\end{aligned}
$$

comprises the soliton mass $M_{0}$ of order $N_{C}$, the pionic rotational energy of order $N_{C}^{-1}\left(\Theta_{\pi}\right.$ pionic moment of inertia) and the kaonic rotational energy of order $N_{C}^{0}\left(\Theta_{K}\right.$ kaonic moment of inertia). The non-trivial $N_{C}$ assignment to the kaonic rotational energy is caused by the WZW term which selects the lowest lying multiplet depending on the number of colors. For odd $N_{C}$ the "nucleon" with spin and isospin $1 / 2$ and hypercharge $N_{C} / 3$ sits in the multiplet with the labels

$$
(p, q)=\left(1, \frac{N_{C}-1}{2}\right), \quad C_{2}=\frac{N_{C}^{2}}{12}+\frac{N_{C}}{2}+\frac{3}{4} .
$$

With that eigenvalue $C_{2}$ of the Casimir operator Eq. (5) is immediately verified.

For the 1-loop calculation fluctuations $\eta_{a}$ are introduced through the ansatz

$U=A \sqrt{U_{0}} e^{i \lambda_{a} \eta_{a} / f} \sqrt{U_{0}} A^{\dagger}, \quad a=1, \ldots, 8$,

and the corresponding equations of motion (e.o.m.) which according to their time dependence $\sim e^{-i \omega t}$ may be written as

$h_{a b}^{2} \eta_{b}=\omega^{2} n_{a b}^{2} \eta_{b}$

( $h_{a b}^{2}$ is a differential operator and $n_{a b}^{2}$ the metric) have to be solved for the phase-shifts. Because the e.o.m. (8) decouple for the different meson species into partial waves characterized by phonon spin $L$ and parity the pionic, kaonic and eta phase-shifts may be summed up separately over the various channels $(L c)$

$$
\delta^{x}(p)=\sum_{L c}(2 L+1) \delta_{L c}^{x}(p), \quad x=\pi, K, \eta .
$$

The ultra-violet divergencies contained in the Casimir energy are related to the high momentum behaviour of these phaseshifts

$$
\delta^{x}(p) \stackrel{p \rightarrow \infty}{\rightarrow} a_{0}^{x} p^{3}+a_{1}^{x} p+\frac{a_{2}^{x}}{p}+\cdots,
$$

with expansion coefficients $a_{0}^{x}, a_{1}^{x}, a_{2}^{x}$ known analytically for the $\mathrm{N} \ell \sigma$ model (the explicitly denoted terms give rise to at least logarithmically divergent expressions). These coefficients obey the important $\mathrm{ChO} 4$ relation

$$
\begin{aligned}
\sum_{x} & {\left[3 \pi m_{x}^{4} a_{0}^{x}-4 \pi m_{x}^{2} a_{1}^{x}+8 \pi a_{2}^{x}\right] } \\
= & \sum_{i=1}^{8} \Gamma_{i} \int d^{3} r \mathscr{L}_{i}^{(4)},
\end{aligned}
$$

where the $\Gamma_{i}$ 's are simple numerical factors given in [6] and which is used below for regularisation of the Casimir energy. For the full model (2) the coefficients have to be determined numerically and the challenge is to calculate the phase-shifts with great precision up to $p_{\max } \simeq 25 m_{\pi}$ where $L_{\text {max }} \simeq 100$ partial waves are needed (for details see [2]). With these informations at hand the divergencies in the 1-loop contribution may be isolated using dimensional regularisation

$$
\begin{aligned}
E_{\mathrm{cas}}= & \frac{1}{2 \pi} \sum_{x}\left(-\int_{0}^{\infty} \frac{p d p}{\sqrt{p^{2}+m_{x}^{2}}}\right. \\
& \times\left[\delta^{x}(p)-a_{0}^{x} p^{3}-a_{1}^{x} p-\frac{a_{2}^{x}}{p}\right] \\
& -m_{x} \delta^{x}(0) \\
& +\frac{3 m_{x}^{4} a_{0}^{x}}{16}\left(\frac{1}{6}+\ln \frac{m_{x}^{2}}{\mu^{2}}\right)-\frac{m_{x}^{2} a_{1}^{x}}{4} \ln \frac{m_{x}^{2}}{\mu^{2}} \\
& \left.+\frac{a_{2}^{x}}{2}\left(1+\ln \frac{m_{x}^{2}}{\mu^{2}}\right)\right) \\
& +\Lambda(\mu) \sum_{x}\left[3 \pi m_{x}^{4} a_{0}^{x}-4 \pi m_{x}^{2} a_{1}^{x}+8 \pi a_{2}^{x}\right] \\
\equiv & \sum_{x} E_{\mathrm{cas}}^{x}(\mu) \\
& +\Lambda(\mu)\left[\sum_{i=1}^{8} \Gamma_{i} \int d^{3} r \mathscr{L}_{i}^{(4)}+\operatorname{higher} \mathrm{ChOs}\right],
\end{aligned}
$$


which involves a scale $\mu$ to render the arguments in the logarithms dimensionless. The divergencies as $d \rightarrow 4$ reside in

$$
\begin{aligned}
\Lambda(\mu)= & \frac{\mu^{d-4}}{16 \pi^{2}} \\
& \times\left[\frac{1}{d-4}-\frac{1}{2}\left(\Gamma^{\prime}(1)+\ln (4 \pi)+1\right)\right],
\end{aligned}
$$

and may finally be absorbed into a redefinition of the LECs

$L_{i}^{r}(\mu)=L_{i}-\Gamma_{i} \Lambda(\mu)$,

$L_{i}^{r}(\mu)=L_{i}^{r}\left(m_{\varrho}\right)-\frac{\Gamma_{i}}{32 \pi^{2}} \ln \left(\frac{\mu^{2}}{m_{\varrho}^{2}}\right)$,

which become scale-dependent. The renormalisation scheme is identical to that used in chiral perturbation theory.

From (12) it is also noticed that the regularisation scheme must fail for $m_{K} \gg m_{\pi}$ : in the limit $m_{K} \rightarrow \infty$ we would obtain an infinite contribution from the second row containing the chiral logarithms (the term $m_{K} \delta^{K}(0) / 2 \pi$ would cancel the bound state contribution $\frac{1}{2} \sum_{z} \omega_{z}$ which has to be added in that case because the infinitesimal kaonic rotations appear at finite energies).

For $m_{K}=m_{\pi}$ with the finite contributions in (12) the nucleon mass in tree +1 -loop is finally determined

$$
\begin{aligned}
E_{\text {tree + 1-loop }}= & M_{0}(\mu)+\frac{3}{8 \Theta_{\pi}(\mu)}+\frac{N_{C}}{4 \Theta_{K}(\mu)} \\
& +\sum_{x} E_{\text {cas }}^{x}(\mu) .
\end{aligned}
$$

All quantities involved become scale-dependent in a non-trivial way, this will be investigated. Because the contributions of the various mesons enter additively we may consider them separately.

\subsection{Pions}

The pionic contribution is the same as in $\mathrm{SU}(2)$, this was mentioned already. In Ref. [2] we obtained a 1-loop contribution $E_{\text {cas }}^{\pi}\left(m_{Q}\right)=-680 \mathrm{MeV}$ at scale $\mu=m_{\varrho}$. Further it was found that the scale-dependences of $M_{0}(\mu)$ and $E_{\text {cas }}^{\pi}(\mu)$ cancel almost exactly over a wide region of scales, compare Fig. 3.2 in that reference. This finding was interpreted as strong evidence for the reliability of the renormalisation procedure and also for the reasonable choice of the effective Skyrme parameter $e$. The pionic rotational energy in (5) is very small by itself and its scale-dependence is relatively weak such that it does not destroy this property.

\subsection{Eta}

The coupling of the $\eta$ to the soliton proceeds through the mass terms only and consequently is extremely weak. The resulting 1-loop contribution $E_{\text {cas }}^{\eta}\left(m_{\varrho}\right)=+0.5 \mathrm{MeV}$ is tiny.

\subsection{Kaons}

Because in the kaonic sector we have four infinitesimal kaonic rotations the phase-shift starts at $\delta^{K}(0)=4 \pi$ according to Levinson's theorem. This fact, although the zero-modes do not contribute by themselves (because they are located at zero energy), leads to a large negative Casimir energy which at scale $\mu=m_{\varrho}$ amounts to $E_{\text {cas }}^{K}\left(m_{Q}\right)=-425 \mathrm{MeV}$. This contribution compensates nicely for the kaonic

Table 1

Nucleon mass in SU(2) and symmetric SU(3) with and without

\begin{tabular}{|c|c|c|c|c|}
\hline & & SU(2) & $\begin{array}{l}\text { SU(3) } \\
\text { without } \\
\text { WZW }\end{array}$ & $\begin{array}{l}\text { SU(3) } \\
\text { with } \\
\text { WZW }\end{array}$ \\
\hline soliton mass & $N_{C}^{1}$ & 1630 & 1630 & 1630 \\
\hline pionic rotation & $N_{C}^{-1}$ & 70 & 70 & 70 \\
\hline kaonic rotation & $N_{C}^{0}$ & - & 390 & 350 \\
\hline total tree & & 1700 & 2090 & 2050 \\
\hline$\pi$ 1-loop & $N_{C}^{0}$ & -680 & -680 & -680 \\
\hline$K$ 1-loop & $N_{C}^{0}$ & - & -425 & -600 \\
\hline$\eta$ 1-loop & $N_{C}^{0}$ & - & +0.5 & +0.5 \\
\hline total tree +1 -loop & & 1020 & 985 & 770 \\
\hline
\end{tabular}
WZW term. The individual tree and 1-loop contributions are listed separately and their $N_{C}$ order is indicated. All energies are given in $\mathrm{MeV}$ 


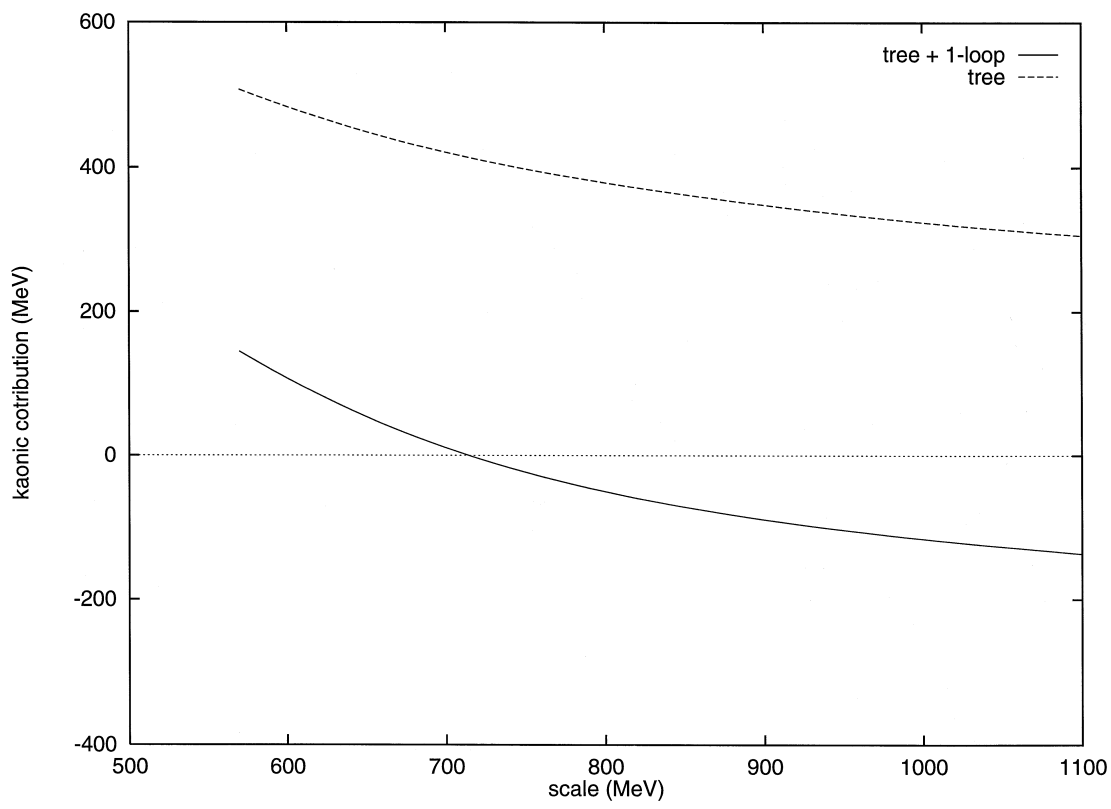

Fig. 1. Scale-dependence of the kaonic contributions without WZW in tree (dashed) and tree + 1-loop (solid). The inclusion of the 1-loop contribution amplifies the scale-dependence.

rotational energy $N_{C} / 4 \Theta_{K}\left(m_{o}\right)=+390 \mathrm{MeV}$ which is of the same order $N_{C}^{0}$ (Table 1). However, the scale-dependence of the rotational energy is enhanced further if the 1-loop contribution is included as is noticed from Fig. 1. This indicates that there is an important term missing.

\subsection{Kaons with WZW term}

Inclusion of the WZW term has two effects (i) it adds a contribution to the kaonic moment of inertia and (ii) it modifies the e.o.m. for the fluctuations (8) introducing a term linar in $\omega$.

\section{- Kaonic moment of inertia}

The WZW term provides the driving term for induced soliton components $\eta_{a}$ proportional to the kaonic angular velocities $\Omega_{e}[8]$

$$
h_{a b}^{2} \eta_{b}=\frac{N_{C}}{2 \sqrt{3}} \frac{\sqrt{\Theta_{K}}}{2 f_{K}} B_{0} f_{8 a b} z_{b}^{e} \Omega_{e}, \quad a, b, e=4-7,
$$

which finally lead to a contribution to the moment of inertia. Here, $h_{a b}^{2}$ represents the differen- tial operator encountered in (8), $B_{0}$ is the baryon density and $z_{a}^{e}$ are the kaonic rotational zeromodes defined below. For $m_{K}=m_{\pi}$ Eq. (16) does not possess a unique solution, because the zero-modes as solutions of the homogeneous equation can always be added with arbitrary strength. This pecularity is caused by the introduction of supernumerary variables through the collective rotations which causes a linear dependence of the angular momenta on the momentum fields conjugate to $\eta_{a}$ (primary constraints). In order to avoid double counting and also to make the Legendre transformation to the hamiltonian well defined [9], we have to impose the constraints

$$
\int d^{3} r z_{a}^{e} n_{a b}^{2} \eta_{b}=0, \quad z_{a}^{e}=\frac{2 f_{K}}{\sqrt{\Theta_{K}}} \sin \left(\frac{F}{2}\right) f_{a e i} \hat{r}_{i}
$$

that the induced components $\eta_{a}$ be orthogonal to the infinitesimal rotations (secondary constraints). These constraints have to be added with multipli- 
ers $\lambda_{e}$ to the lagrangian and lead to a modification of Eq. (16)

$$
\begin{aligned}
& h_{a b}^{2} \eta_{b}=\frac{N_{C}}{2 \sqrt{3}} \frac{\sqrt{\Theta_{K}}}{2 f_{K}} B_{0} f_{8 a b} z_{b}^{e} \Omega_{e}+\lambda_{e} n_{a b}^{2} z_{b}^{e}, \\
& \lambda_{e}=\frac{N_{C}}{4 \sqrt{3}} \frac{1}{\sqrt{\Theta_{K}}} f_{8 d e} \Omega_{d},
\end{aligned}
$$

which is then free of spurious zero-mode components. Instead of going through Dirac's quantization procedure [9], we may as well reinsert the induced components into the lagrangian and read off the moment of inertia from the terms proportional to $\Omega^{2}$. For $m_{K}=m_{\pi}$ and scale $\mu=m_{o}$ we obtain $\Theta_{K}\left(m_{\varrho}\right)=(1.92+0.21) \mathrm{GeV}^{-1}=2.13$ $\mathrm{GeV}^{-1}$ a $10 \%$ contribution from the induced component. Of course also for $m_{K} \neq m_{\pi}$ the constraints (17) have to be implemented, but there the induced component decays very rapidly with increasing kaon mass justifying that this contribution is normally neglected. In our case it helps to soften the scale-dependence of the kaonic rotational energy as is noticed by comparing Fig. 1 with Fig. 2.
- Phase-shifts

The WZW term introduces a term linear in $\omega$ into the e.o.m. (8)

$h_{a b}^{2} \eta_{b}+i \omega \frac{N_{C} B_{0}}{\sqrt{3} f_{K}^{2}} f_{8 a b} \eta_{b}=\omega^{2} n_{a b}^{2} \eta_{b}$.

As a consequence not all infinitesimal rotations remain at zero energy. For physical kaon mass, two of them appear as bound states and are interpreted as kaonic excitations of the nucleon (bound-state approach [5]). For $m_{K}=m_{\pi}$ these states appear as resonances in the continuum and consequently the kaonic phase-shift starts only at $\delta^{K}(0)=2 \pi$. Again, because these states are already taken into account by the kaonic collective rotations we have to impose the constraints (17) in order to project the zero-modes from the space of allowed fluctuations

$$
h_{a b}^{2} \eta_{b}+i \omega \frac{N_{C} B_{0}}{\sqrt{3} f_{K}^{2}} f_{8 a b} \eta_{b}=\omega^{2} n_{a b}^{2} \eta_{b}+\lambda_{e} n_{a b}^{2} z_{b}^{e}
$$

(with lagrangian multipliers determined from (17)). The same procedure had to be applied also

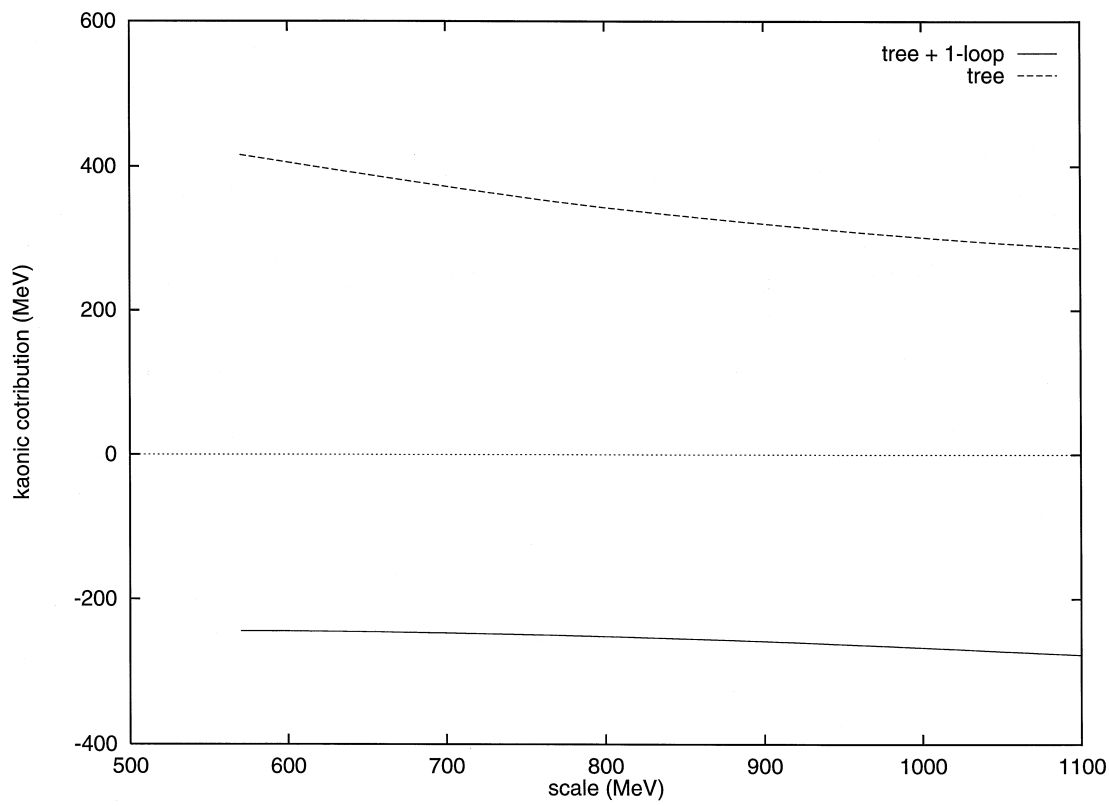

Fig. 2. Same as Fig. 1 but with the WZW term considered. The tree +1 -loop contribution is almost scale-independent over a wide region of scales. 
in [2], where it were external fields that destroyed the rotational symmetry. The phase-shift calculated from (20) starts again at $\delta^{K}(0)=4 \pi$ as it should and the Casimir energy may as usual be computed with the phase-shift formula according to (12). Alternatively, and of course with the same result, the linear term in $\omega$ may be integrated from the path integral (compare [2], Eq. (2.34)) and may be replaced by an additional potential in the scattering Eqs. (20).

With inclusion of the WZW term the Casimir energy $E_{\mathrm{cas}}^{K}\left(m_{\varrho}\right)=-600 \mathrm{MeV}$ at scale $\mu=m_{\varrho}$ over-compensates the kaonic rotational energy $N_{C} / 4 \Theta_{K}\left(m_{\varrho}\right)=+350 \mathrm{MeV}$ (Table 1). In contrast to the case without WZW the scale-dependences of these quantities are opposite such that the kaonic energy in tree +1 -loop becomes almost scale-independent over a wide region (Fig. 2) quite similar to the pionic contributions (for very small scales the soliton becomes unstable due to a too strong symmetric $\mathrm{ChO} 4$ term). This confirms that the WZW term plays an important role also in this context.

\section{Results}

Table 1 comprises the results for the nucleon mass in the SU(3) symmetric chiral soliton model with and without the WZW term taken into account. It should be kept in mind that there are no additional adjustable parameters in the game, the effective Skyrme parameter was taken as in the SU(2) calculation. It is noticed that in both cases the tree mass of $\simeq 2 \mathrm{GeV}$ is appreciably reduced into the region of the physical nucleon mass. This is compatible with $N_{C}$ counting because for the kaonic contributions tree and 1-loop are of the same order $N_{C}^{0}$ and because a strong cancellation between the two occurs. As was discussed in the previous section, scale-independence requires the inclusion of the WZW term. Thus, the results for the nucleon mass are in case of SU(3) symmetry $770 \mathrm{MeV}$ and for $\mathrm{SU}(2) 1020 \mathrm{MeV}$ such that the empirical nucleon mass actually lies in between these two limiting values. Once the lagrangian is fixed the calculation presented is exact to order $N_{C}^{0}$, there are no other contributions to this order. Unfortunately we were not in the position to calculate 1-loop corrections for finite $m_{K} \neq m_{\pi}$ for the reasons discussed in the introduction. Possibly one can estimate the Casimir energy for $m_{K} \gtrsim m_{\pi}$ in the rotator approach where one finds Euler angle dependent scattering equations [10], but it is difficult to control the necessary approximations (neglection of the terms linear in the fluctuations which arise from the rotating hedgehog being not an exact solution). On the other hand for $m_{K} \gg m_{\pi}$ the 1-loop contributions could possibly be calculated using the bound-state approach [5] which also remains to be investigated.

\section{Acknowledgements}

This work is supported by JNICT, Portugal (Contract PRAXIS $/ 2 / 2.1 /$ FIS $/ 451 / 94)$. The author would like to thank G. Holzwarth and N. Scoccola for helpful discussions.

\section{References}

[1] B. Moussallam, Ann. Phys. 225 (1993) 264.

[2] F. Meier, H. Walliser, Phys. Rep. 289 (1997) 383.

[3] B. Schwesinger, H. Weigel, Phys. Lett. B 267 (1991) 438.

[4] H. Yabu, K. Ando, Nucl. Phys. B 301 (1988) 601.

[5] C.G. Callan, K. Hornbostel, I. Klebanov, Phys. Lett. B 202 (1988) 269.

[6] J. Gasser, H. Leutwyler, Nucl. Phys. B 250 (1985) 465.

[7] G. Ecker, Czech. J. of Phys. 44 (1995) 405.

[8] H. Weigel, J. Schechter, N.W. Park, U.G. Meissner, Phys. Rev. D 42 (1990) 3177.

[9] P.A.M. Dirac, Lectures on Quantum Mechanics, Belfer Graduate School of Science, 1964.

[10] B. Schwesinger, Nucl. Phys. A 537 (1992) 253. 